INPLASY

PROTOCOL

To cite: Lin et al. Goal-directed therapy for aneurysmal subarachnoid hemorrhage:A systematic review and metaanalysis. Inplasy protocol 202170019. doi:

10.37766/inplasy2021.7.0019

Received: 08 July 2021

Published: 08 July 2021

Corresponding author:

Lu Zhongsheng

LZS13997154047@163.com

Author Affiliation:

Qinghai Provincial People's

Hospital

Support: None.

Review Stage at time of this submission: Formal screening of search results against

eligibility criteria.

Conflicts of interest:

None declared.

\section{Goal-directed therapy for aneurysmal subarachnoid hemorrhage: A systematic review and meta-analysis}

Lin, S1; Zhu, Y2; Lu, Z3.

Review question / Objective: To evaluate the effect of goaldirected therapy on complications and prognosis in patients with ASAH. Effects of goal-directed therapy on complications and prognosis in patients with ASAH.

Condition being studied: Aneurysmal subarachnoid hemorrhage.

Information sources: We conducted a comprehensive search of several authoritative electronic databases, including PubMed, Embase, Cochrane Library, and Web of Science. Key search terms included "Goal Directed Therapy" and "Aneurysmal Subarachnoid Hemorrhage". All journal papers published through July 2021 are included. No other qualifiers were set in the search process, and the search language was limited to English only. In order to avoid omission, references of each study were consulted at the same time.

INPLASY registration number: This protocol was registered with the International Platform of Registered Systematic Review and Meta-Analysis Protocols (INPLASY) on 08 July 2021 and was last updated on 08 July 2021 (registration number INPLASY202170019).

\section{INTRODUCTION}

Review question / Objective: To evaluate the effect of goal-directed therapy on complications and prognosis in patients with ASAH. Effects of goal-directed therapy on complications and prognosis in patients with ASAH.
Rationale: Aneurysmal subarachnoid hemorrhage (aSAH) is a nervous system disease with high morbidity and mortality. The cerebral vasospasm (CVS) after aSAH and its secondary injuries, such as delayed cerebral ischemia (DCl), cerebral infarction and other neurological deficits, are always important factors affecting the prognosis of 
patients. It is very important for aSAH patients with hemodynamic management In the clinical.But at the moment, fluid and hemodynamic assessments are mostly based on clinical judgments and it Has been shown to be insufficient to guide the hemodynamic management of aSAH. Recently, some studies have been conducted on goal-directed therapy (GDT) in patients with aSAH under the guidance of transpulmonary thermodilution monitoring (PICCO), noninvasive cardiac output monitoring (NICOM) devices, or transesophageal echocardiography (TEE) and other relevant hemodynamic monitoring methods. But the results of studies have been mixed. As for whether GDT can achieve better outcome and prognosis in the treatment of aSAH patients, there is no conclusive conclusion at present.

Condition being studied: Aneurysmal subarachnoid hemorrhage.

\section{METHODS}

Participant or population: Patients with aneurysmal subarachnoid hemorrhage.

Intervention: Goal-directed therapy.

Comparator: Patients with aneurysmal subarachnoid hemorrhage treated with standard therapy.

Study designs to be included: Randomized controlled trials and prospective cohort studies.

Eligibility criteria: To qualify for a metaanalysis, studies must be randomized controlled trials (RCTs) or cohort studies (prospective) and have 2 groups of patients: one group receiving goal-directed therapy and the other group receiving standard care as usual. For both groups, the study needed to report the main outcomes of interest - cerebral vasospasm (CVS), The prognosis is 3 monthsprognosis, Delayed cerebral ischemia (DCI), and cerebral infarction - by reporting the number of patients with each outcome in each group.
Information sources: We conducted a comprehensive search of several authoritative electronic databases, including PubMed, Embase, Cochrane Library, and Web of Science. Key search terms included "Goal Directed Therapy" and "Aneurysmal Subarachnoid Hemorrhage". All journal papers published through July 2021 are included. No other qualifiers were set in the search process, and the search language was limited to English only. In order to avoid omission, references of each study were consulted at the same time.

Main outcome(s): Cerebral vasospasm (CVS), The prognosis is 3 months, Delayed cerebral ischemia(DCI), and cerebral infarction.

Quality assessment / Risk of bias analysis: The quality of the included RCTs was evaluated using the Cochrane Systematic Evaluation Manual criteria, including random method, blind method, allocation concealment, data integrity, selective reporting, and other offset; A NOS scale was used to evaluate the quality of the included non-RCTs, including selection, comparability, exposure, or outcome.

Strategy of data synthesis: Statase /16.0 software was used for statistical analysis of the data. The odds ratio (OR) was used as the effect value index for the dichotomous variables. Heterogeneity was determined based on the chi-square test results of heterogeneity and 12 value. If the chi-square test results $\mathrm{P}>0.05$ and $12<$ $50 \%$, the fixed effect model was used to combine the effect values. Otherwise, the random effects model is used to combine the effect values. $P<0.05$, the difference was considered statistically significant. Funnel plots and Begger's and Egger's tests were used to show the publication bias of the studies included in the metaanalysis.

Subgroup analysis: None.

Sensitivity analysis: Sensitivity analysis of the combination of effect sizes of each 
outcome indicator was completed in the software Stata/SE 16.0.

Language: No restriction.

Country(ies) involved: China.

Keywords: Aneurysmal Subarachnoid Hemorrhage, Goal-Directed Therapy, Hemodynamic monitoring,Clinical curative effect, meta-analysis.

Dissemination plans: Publication in a peerreviewed journal.

Contributions of each author:

Author 1 - Lin Shengwu.

Email: linshengwu7@163.com

Author 2 - Zhu Yufeng.

Email: 18895318990@163.com

Author 3 - Lu Zhongsheng.

Email: LZS13997154047@163.com 